\title{
THE CORRELATION BETWEEN THE STATISTICAL PASS-BY INDEX VALUES AND THE TOTAL NUMBER OF VEHICLE PASSES
}

\author{
Martin DECKÝ ${ }^{1}$, Eva REMIŠOVÁ ${ }^{1}$, Matej BRNA ${ }^{1, *}$, Marek DRLIČIAK ${ }^{1}$, Matúš \\ KOVÁČ 1
}

${ }^{1}$ Department of Highway and Environmental Engineering, University of Zilina, Univerzitna 8215/1, Zilina 01026, Slovakia.

corresponding author: matej.brna@uniza.sk

\begin{abstract}
In this study, the traffic noise degradation in asphalt pavements was analysed using the 'Statistical Pass-By method'. The sound levels of two surfaces were monitored during 9 and 12 years of service, respectively. By comparing the dependencies of the maximum Aweighted sound pressure level on logarithm of vehicle velocity, an increase in the sound level was found at all recorded speeds. Following an analysis of sound levels, as combined with the statistical pass-by index (SPBI) calculated versus age (expressed in vehicles), it was determined that the noise is an increasing power function of SPBI values on vehicle passes, based on an approximation of noise level adjustment to a reference temperature of $20^{\circ} \mathrm{C}$ (using a coefficient of 0.06 for asphalt concrete surface AC11 and -0.03 for mastic asphalt SMA11). The adjusted traffic noise degradation model showed that the SMA11 surface has a higher resistance to acoustic degradation than AC11 surface.
\end{abstract}

Keywords:

Traffic;

Noise;

SPBI;

Asphalt surface;

Ageing.

\section{Introduction}

Road planning considers the demands of present and future users, road agencies, and society to assure optimum structural performance, serviceability, and sustainability, while minimising the longterm financial and environmental costs. Road users expect traffic safety, driving comfort, economical travel, and environmentally friendly services. These expectations imply performance-related requirements on road pavement surfaces, such as skid resistance, evenness, and homogeneity without distress, along with low noise and pollution emissions [1, 2].

The performance-related and safety characteristics of a surface change owing to traffic loading and environmental effects; so, do its acoustic properties. Some authors [3-7] have conducted acoustic assessments on different surfaces with different characteristics, e.g., based on surface texture (texture depth), unevenness, and distress (no distress, cracking, alligator cracking, and ravelling), under different traffic flow conditions (vehicle type, speed), and by different measurement methods. The results were thus influenced by several factors, and are difficult to compare with each other. In another study [8], the spectral compositions of close-proximity results measured on an asphalt surface over two years did not show clear results, owing to the repeatability of the measurements, i.e., the same track, lateral direction (right or left track), speed, direction, and a number of lanes. In contrast, the 'Statistical Pass-By method' (used in the authors' research) seems more suitable for the long-term monitoring of the acoustic properties of surfaces. The acoustic performance of road surfaces expressed by time dependence is presented in the literature [9] as linear, logarithmic, or exponential functions with an increase between 0.03 and $5 \mathrm{~dB}$ per year. The increase in tyre/road noise levels in time is very different among the different surfaces (mixture type in wearing course), traffic loading, and climatic conditions. Logarithmic regression model by [10] of noise level on a combination of a set of factors (pavement type, traffic data - heavy traffic, climatic conditions - annual precipitation, number of freeze-thaw cycles, number of ice days) shows a strong impact of climatic factors on acoustical 
ageing. However, the acoustic ageing trends of two sites with similar climatic conditions are significantly different. The noise level decreased with increasing temperature at a rate of $-0.1 \mathrm{~dB} /{ }^{\circ} \mathrm{C}$ for dense asphalt pavements while $-0.06 \mathrm{~dB} /{ }^{\circ} \mathrm{C}$ for porous pavements [11]. When evaluating the longterm monitoring of the acoustic performance of asphalt surfaces, it is important to compare under similar conditions. Therefore, the measurements are converted to the reference temperature $+20^{\circ} \mathrm{C}$ by default. Nevertheless, there remains a lack of knowledge on the acoustical ageing/degradation of surfaces depending on age (number of years/months) and/or traffic load (number of vehicles passing). Therefore, this research is focused on the long-term monitoring of noise emissions from different asphalt surfaces under the same climatic conditions, from the start of their service (wearing course production) during 9 and 12 years. Thus, it will be possible to obtain a model for the degradation of the acoustic properties of surfaces, and the results of this research can be used to design road pavements with long-term performance, and durable and effective noise features.

The relevant factors in the case of the road pavement affecting acoustic descriptors in the environment [12] are the surface texture, type and air void characteristics of the wearing course, in interaction with the type/condition of the vehicle tyres and vehicle speed. Part of tyre/road noise is absorbed by the surface, depending on the porosity of the surface material. Just the interconnection of air gaps in a material is acoustically relevant. However, all surfaces in use lose their initial noise properties due to traffic load (compaction and reducing porosity by vehicle passes) and climatic impacts (clogging by the dirt). Therefore, it is desirable to retain noise reduction performance for a long time [13]. Studies have been conducted on surface materials interacting with the rolling of vehicle wheels aiming to generate less acoustic energy, and suitable so-called 'low-noise surface' solutions have been proposed [5, 14-16]. The performance of low noise surfaces in time shows faster acoustic and structural ageing comparing with traditional asphalt surfaces. After 3 years of service, the sound level $\left(L_{A \max }\right)$ of the low-noise surface from very thin asphalt concrete and porous asphalt concrete increased by 5.7 to $6.5 \mathrm{~dB}$ to a level similar to the standard asphalt concrete [17].

The reduction of tyre noise is a priority in many countries, either to ease the discomfort of motorists, or to protect the interests of nearby residents. Forecasting the acoustic performance of a pavement is one approach to design. The mixture composition now plays an important role, in particular as concerns the possibility of cost savings for additional necessary solutions. The aim of the design of a pavement surface asphalt mixture [18] is to provide sufficient durability and long-term acoustical benefits. As such, the development of an appropriate model can provide an effective way to predict the acoustic properties of road pavements, and their degradations over time.

In the paper, the changes in the noise level of two asphalt wearing courses (AC 11 and SMA 11) over time and traffic load during 9 and 12 years of service are studied. Other relevant factors from the surface point of view as asphalt porosity, surface texture or distresses have not been considered.

\section{Methodology of the experimental traffic noise measurement}

\subsection{Measuring sections}

For the experimental measurement of noise emissions and monitoring of the acoustic aging of the road surface, two experimental road sections in the Slovak Republic, region Žilina, were selected. The measuring sections for the long-term monitoring of noise emissions were chosen so as to determine the effect of the type of the surface on acoustic ageing at different traffic loads, namely, different vehicle intensities, traffic flow compositions, and driving speeds. Both measuring sections were located in the same region Žilina (Fig. 1.) and same climatic region $316 \mathrm{~m}$ above sea level. It was assumed that the influence of the climatic conditions on the surface degradation of both road sections would be the same. 


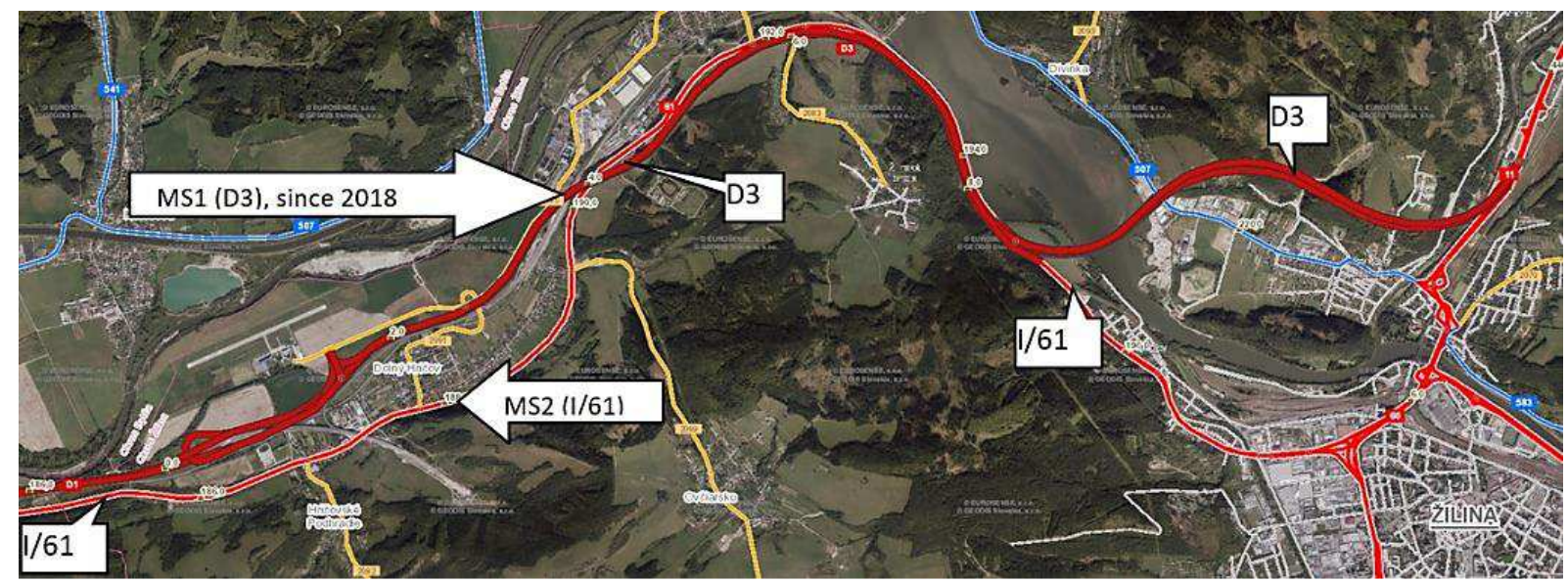

Fig. 1: Localizations of measured sections MS1 and MS 2.

Measuring section 1 (MS1) is located on the trans-regional motorway D3 (part of the European route E75); it was built in the year 2006 in half-profile and in 2008 in full profile (Fig. 2), as a four-lane way with a categorical width of $24.5 \mathrm{~m}$ and maximum permitted vehicle speed of $130 \mathrm{~km} / \mathrm{h}$ (high road speed category). The surface of this highway section was built from an asphalt mixture of stone mastic asphalt, i.e. SMA11.

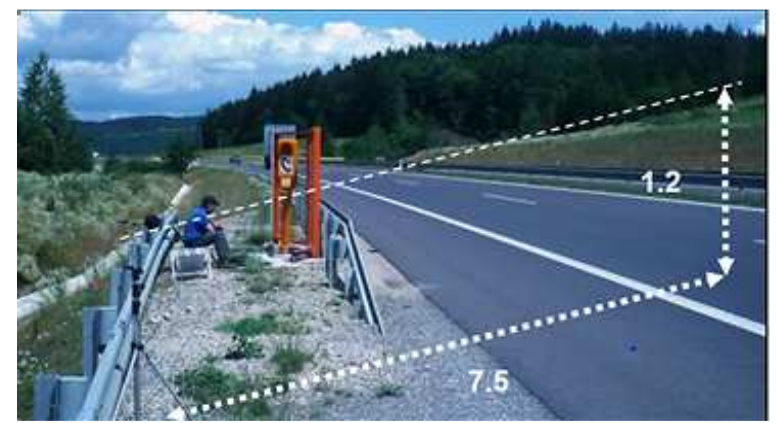

Fig. 2: Measuring section MS1 on motorway D3.

Measuring section 2 (MS2) is situated on the main two-lane road No. 61, with a categorical width of $11.5 \mathrm{~m}$, maximum permitted vehicle speed of $90 \mathrm{~km} / \mathrm{h}$ (medium road speed category), and asphalt pavement construction. The wearing course was built from an asphalt-concrete mixture of type AC 11 (dense asphalt concrete). The asphalt mixture was produced, laid, and compacted in 2008 (Fig. 3).
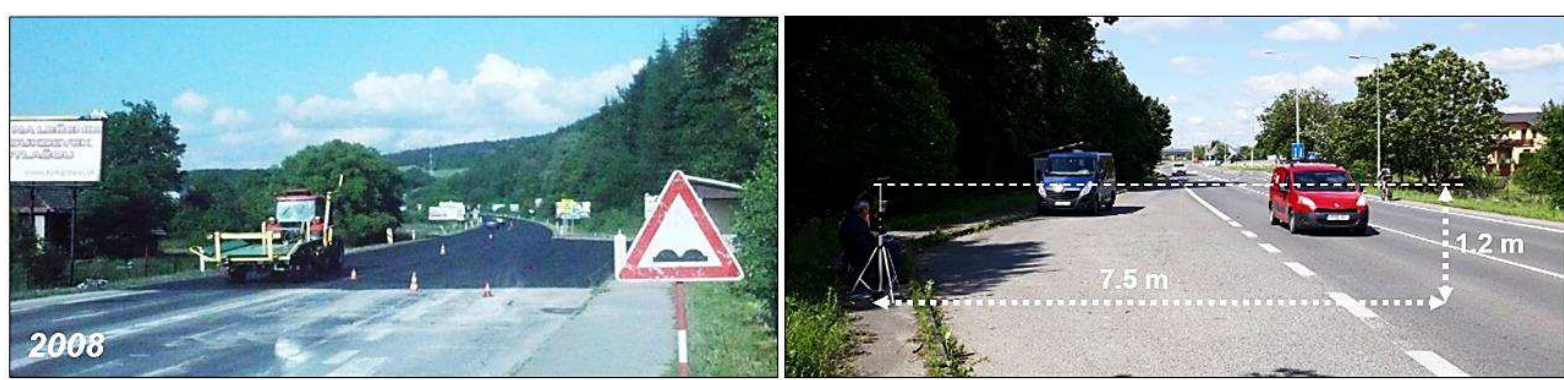

Fig. 3: Views of the measuring section MS2 in 2008 (laying of the asphalt wearing course) and in 2020 (measuring process).

The main reason for using these road sections with these two types of wearing courses, besides the close locations and high volume of traffic, was the fact that the SMA is the most used type of asphalt mixture for wearing course for motorways and the AC for 1st, and 2nd class roads in Slovakia. The appropriateness of the choice of the two types of wearing courses can be also justified by the fact that the average of those two pavements was later selected as the virtual reference 
pavement for the 'Common Noise Assessment Methods in Europe' (CNOSSOS-EU) prediction model [19].

\subsection{Traffic intensity analysis}

The traffic loads on the monitored two sections were obtained from a nationwide road traffic survey in 2015, as shown in Fig. 4. The volumes, expressed by AADT (Annual Average Daily Traffic), correspond to the average loads on the main and trans-regional roads of the Slovak Republic. The commissioning of the highway section in 2008 attracted traffic from the main road No. 61 . The traffic load on the main road No. 61 shows the decrease in 2010. In view of the traffic corridor, which includes highway D3 and road No. 61, the total traffic increases from 1995. The individual car traffic represents mainly local, regional traffic what was found from the region mobility survey. The common indicator for both measuring sections is a significant proportion of heavy traffic $24 \%$. Almost 5,700 heavy vehicles per day (both directions) driving on the highway, representing $80 \%$ of the total number of heavy vehicles in the corridor.

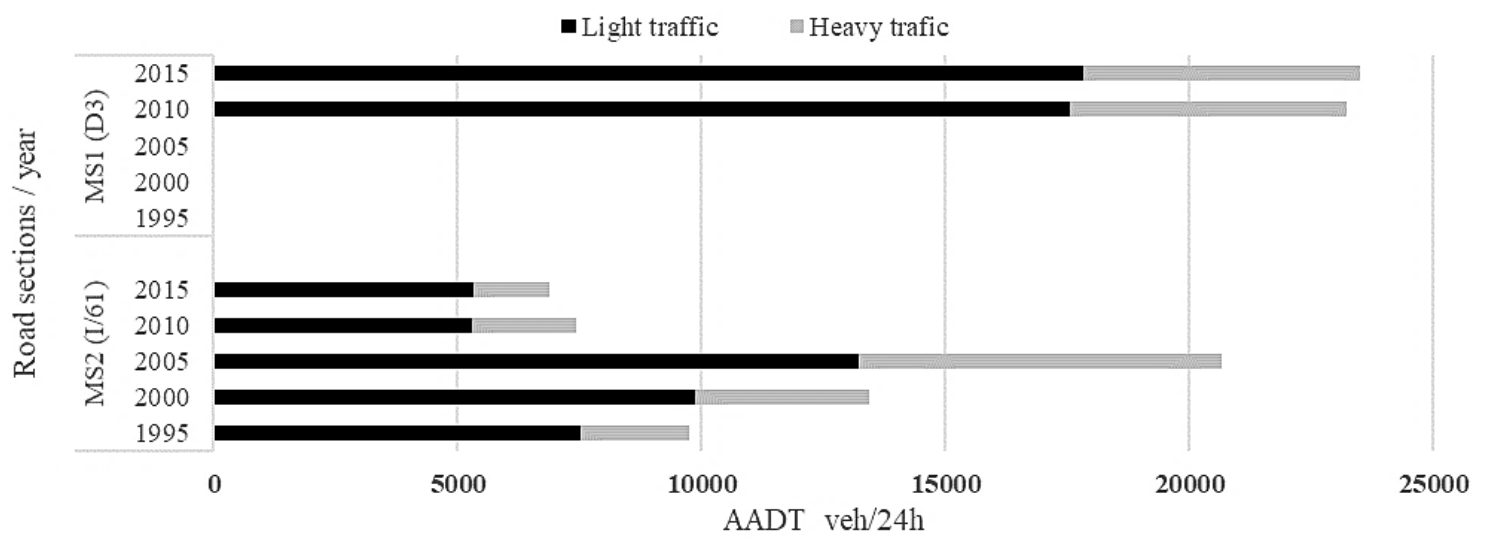

Fig. 4: Traffic development on monitored sections (source: SSC).

\subsection{Methodology}

In this study, the 'Statistical Pass-By method' (SPB), as specified in [20], was used for traffic noise measurements. The measurements were performed every year at the same place from 2008 , using the SPB. The maximum A-weighted sound level $\left(L_{A \max }\right)$ was induced by the vehicle passes at the corresponding driving speeds when passing through the section with the measuring microphone. The noise (as a sound level) of the passing vehicle and vehicle speed at the same time were recorded, and a specific index was calculated.

During the measurements, photo documentation of individual vehicles was performed. Subsequently, the corresponding $L_{\text {Amax }}$ sound levels were assigned to the speed records in the laboratory. The total number of measurements was about 30\% higher than the minimum due to the possibility of excluding outliers (atypical values e.g., vehicle braking, load oscillation, etc.).

For temperature corrections, the average of the temperatures at the beginning and the end of the experiment was considered. When evaluating the long-term monitoring of the acoustic performance of asphalt surfaces, it is important to compare under similar conditions. Therefore, the measured sound pressure levels were converted to the reference temperature of $+20^{\circ} \mathrm{C}$ by default deriving the coefficient with the highest value of the determination, according to [20].

\subsection{Measuring devices}

The Norsonic sound analyser NOR-121 (accuracy Class 0) was used to measure the specific sound level LA. Before the measurements, regular calibration (calibrator NOR N-1251, precision Class 1) was necessary. In addition to the noise measurement, a velocity radar (Bushnell Speedster II) recorded the real-time speed of the passing vehicle, and a weather station (WS888) recorded the climatic conditions. 


\section{Noise measurement results and analysis}

The analysis and assessment considered three vehicle categories:

- No. 1 - cars,

- No. 2 a - dual-axle heavy vehicles,

- No. $2 b$ - multi-axle heavy vehicles.

On measuring section MS1 (on trans-regional motorway D3), the first measurement was performed in the year 2008, three months after laying a new wearing course from the asphalt mixture SMA 11. Fig. 5 compares the regressions for all reference categories of vehicles for the first measurements in the year 2008, and in 2017. The planned measurements for 2020 could not be conducted, owing to the COVID pandemic. The cars' reference speeds were $v_{1}=110 \mathrm{~km} / \mathrm{h}, v_{2 a}=85$ $\mathrm{km} / \mathrm{h}$, and $v_{2 b}=85 \mathrm{~km} / \mathrm{h}$ (see Table 1). During the nine years of measurement, the traffic service on the motorway D3 at section MS1 shows an average increase in the maximum A-weighted sound level by $4.8 \mathrm{~dB}$ in the case of vehicle type No. 1 - cars; $4.2 \mathrm{~dB}$ for vehicle type No. $2 \mathrm{a}$ - dual-axle heavy vehicles; and $3.5 \mathrm{~dB}$ for vehicle type No. $2 \mathrm{~b}$ - multi-axle heavy vehicles. Thus, the highest increase in the average sound level values at the measured speeds (in terms of absolute value) is observed for vehicle type No. 1 - cars $(4.8 \mathrm{~dB})$.
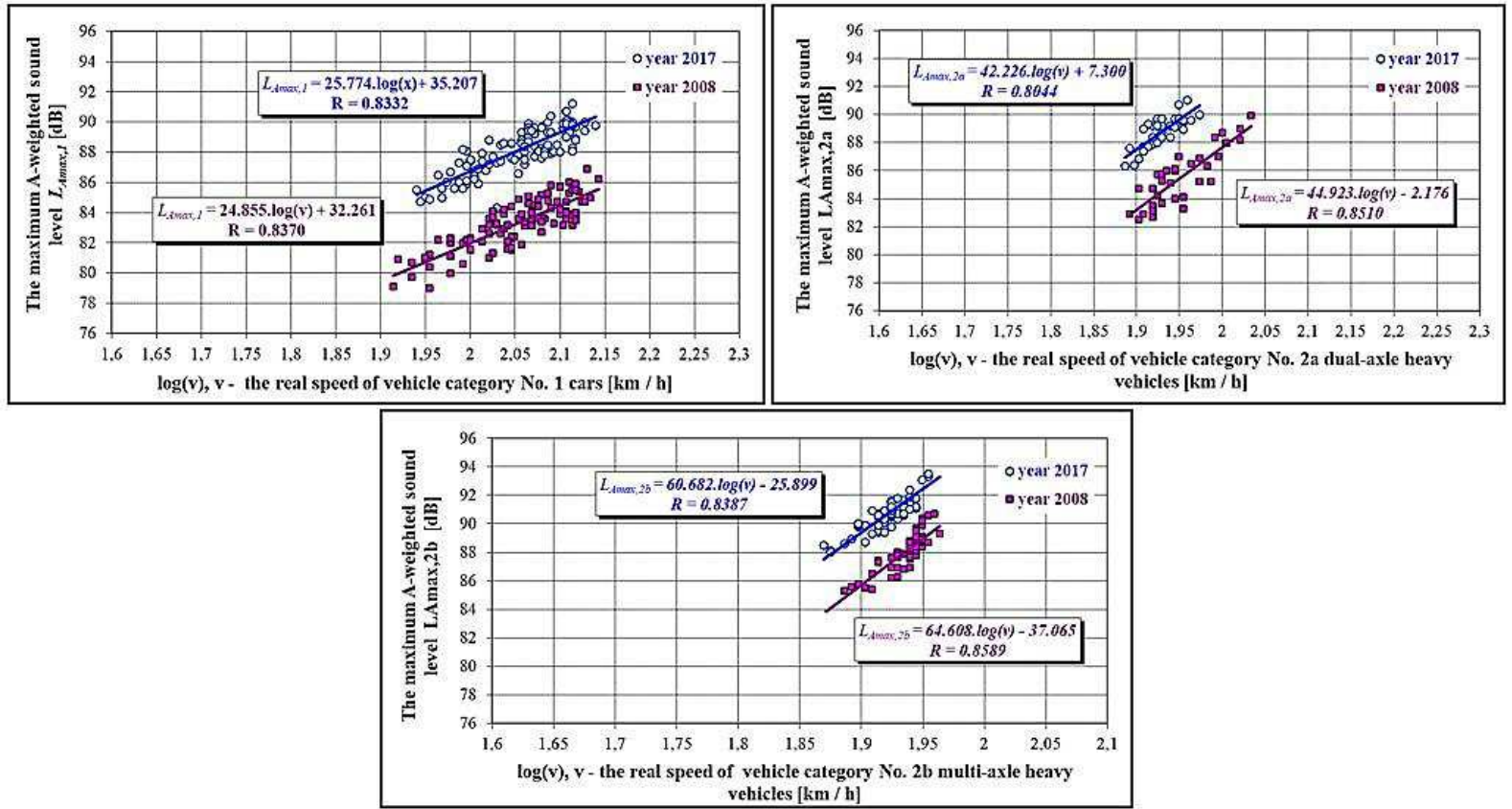

Fig. 5: Regression of the maximum A-weighted sound level and the real vehicle speed of category 1 , $2 \mathrm{a}, 2 \mathrm{~b}$ on MS1.

The results of the regressions for all reference vehicle categories on section MS2, for the measurements between the year 2008 (four months after laying a new wearing course from AC 11) and the year 2020, are shown in Fig. 6 . The cars' reference speeds were $v_{1}=80 \mathrm{~km} / \mathrm{h}, v_{2 a}=70 \mathrm{~km} / \mathrm{h}$, and $v_{2 b}=70 \mathrm{~km} / \mathrm{h}$, see Table 1. During 12 years of traffic service on the main road No. 61 in MS2, there is an average increase in the sound level of $4.3 \mathrm{~dB}$ in the case of No. 1 - cars; a $3.7 \mathrm{~dB}$ increase from vehicle type No. $2 a$ - dual-axle heavy vehicles; and a $2.4 \mathrm{~dB}$ increase from vehicle type No. $2 \mathrm{~b}$ multi-axle heavy vehicles. Thus, the highest increase in the average sound level values at the measured speeds (in terms of absolute value) is observed for vehicle type No. 1 - cars (4.3 dB). 

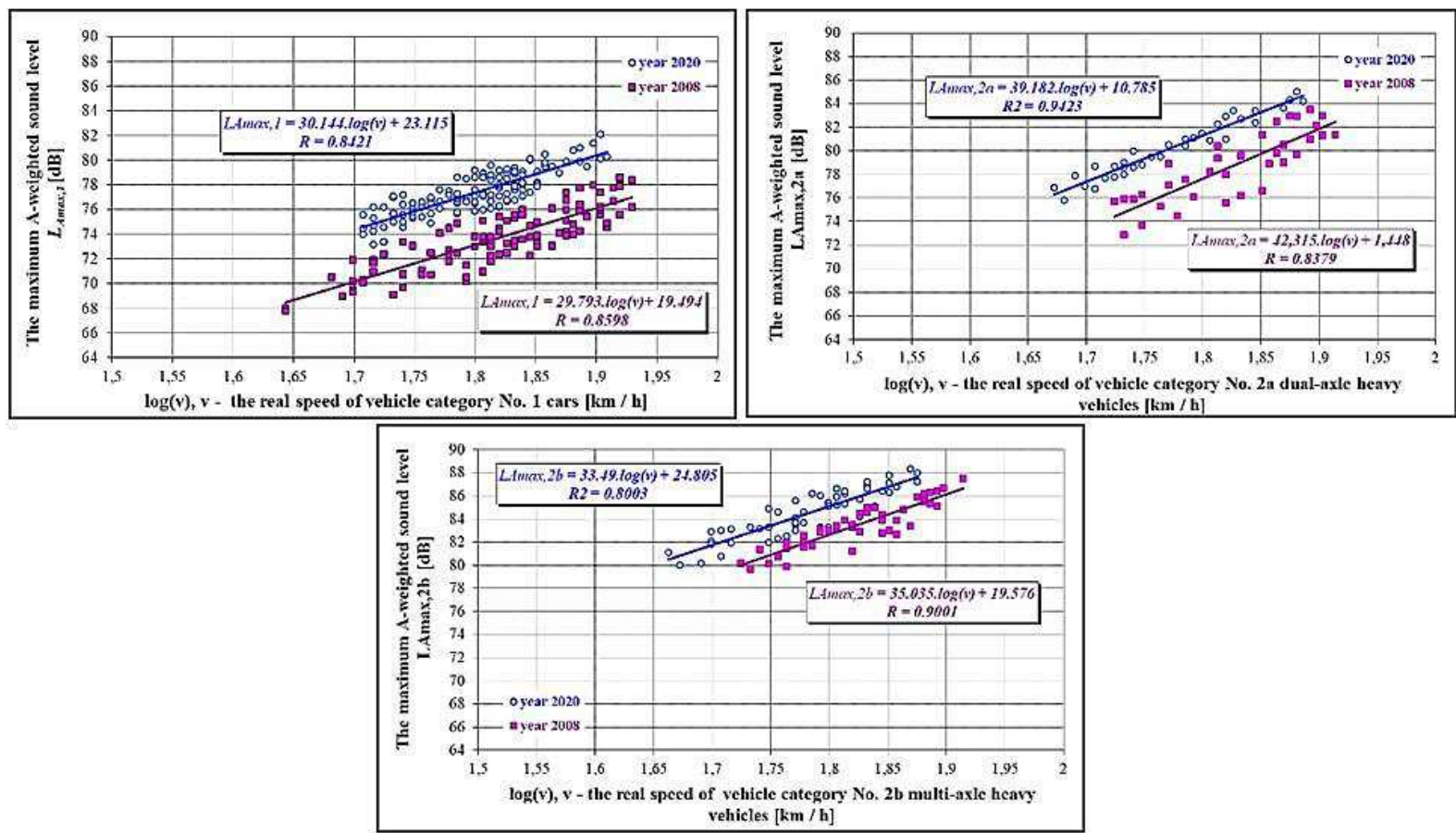

Fig. 6: Regression of the maximum A-weighted sound level and the real vehicle speed of category 1 , $2 \mathrm{a}, 2 \mathrm{~b}$ on MS2.

By comparing the values on the motorway D3 at MS1 with the results on the main road No. 61 at MS2, it can be seen that the increase in the noise exposure on motorway D3 is higher. Thus, the acoustic ageing of the wearing courses of road pavements is caused by traffic loading. The largest difference between the monitored sections MS1 and MS2 is observed when increasing for vehicle category No. 2b, i.e., multi-axle heavy vehicles, at $1.0 \mathrm{~dB}$ (an increase of $3.5 \mathrm{~dB}$ on motorway D3, and an increase of $2.4 \mathrm{~dB}$ on the main road No. 61).

To calculate the SPB index (SPBI), it was necessary to evaluate the regression lines (Fig. 5, Fig. 6), and to determine the sound level $L_{\text {veh }}$ for the reference speeds and vehicle categories. The SPBI allows us to express the complex noise level of the pavement surface for different traffic flows (vehicle combinations), and is calculated as follows:

$S P B I=10 \cdot \log \left[W_{1} \cdot 10^{\wedge}\left(L_{1} / 10\right)+W_{2 a} \cdot\left(v_{1} / v_{2 a}\right) \cdot 10^{\wedge}\left(L_{2 a} / 10\right)+W_{2 b} \cdot\left(v_{1} / v_{2 b}\right) \cdot 10^{\wedge}\left(L_{2 b} / 10\right)\right]$.

In the above, $S P B I$ is the $S P B I[\mathrm{~dB}], L_{1}, L_{2 a}$, and $L_{2 b}$ are the vehicle sound levels for the $1,2 \mathrm{a}$, and $2 \mathrm{~b}$ car categories, respectively $[\mathrm{dB}] ; W_{1}, W_{2 a}$, and $W_{2 b}$ are weighting factors determined by compositions of traffic flow [-], and $v_{1}, v_{2 a}$, and $v_{2 b}$ are the cars' reference speeds $[\mathrm{km} / \mathrm{h}]$. The values for the $S P B I$, including the vehicle categories, reference speeds, sound levels, and weighting factors for measuring sections MS1 (highway D3, high driving speed) and MS2 (main road No. 61, medium driving speed), are shown in Table 1. 
Table 1: Vehicle categories according to EN ISO 11819-1 and results of measurements on measuring sections MS1 and MS2.

\begin{tabular}{|c|c|c|c|c|c|c|c|c|c|c|c|}
\hline \multirow{4}{*}{$\begin{array}{l}\text { Vehicle } \\
\text { category }\end{array}$} & \multirow{4}{*}{\multicolumn{2}{|c|}{ Vehicle characteristics }} & \multicolumn{9}{|c|}{ Number of pass-by and $L_{1}, L_{2 a}, L_{2 b}[\mathrm{~dB}]$} \\
\hline & & & \multirow{3}{*}{ Minimum No. } & \multicolumn{4}{|c|}{ MS1 } & \multicolumn{4}{|c|}{ MS2 } \\
\hline & & & & \multicolumn{2}{|c|}{2008} & \multicolumn{2}{|c|}{2017} & \multicolumn{2}{|c|}{2008} & \multicolumn{2}{|c|}{2020} \\
\hline & & & & No. & $L_{i}$ & No. & $L_{i}$ & No. & $L_{i}$ & No. & $L_{i}$ \\
\hline \multirow{3}{*}{ No. 1} & \multirow{3}{*}{$\begin{array}{c}\text { passenger car } \\
2 \text { axles } \\
4 \text { wheels }\end{array}$} & & 100 & 102 & 83.0 & 104 & 87.8 & 103 & 76.2 & 133 & 80.5 \\
\hline & & & $v_{1}[\mathrm{~km} / \mathrm{h}]$ & \multicolumn{4}{|c|}{110} & \multicolumn{4}{|c|}{80} \\
\hline & & & $W_{1}[-]$ & \multicolumn{4}{|c|}{0.700} & \multicolumn{4}{|c|}{0.800} \\
\hline \multirow{3}{*}{ No. $2 a$} & \multirow{3}{*}{$\begin{array}{l}\text { heavy vehicle } \\
2 \text { axles } \\
\text { above } 4 \text { wheels }\end{array}$} & & 30 & 36 & 84.5 & 36 & 88.8 & 35 & 79.6 & 35 & 83.1 \\
\hline & & & $v_{2 a}[\mathrm{~km} / \mathrm{h}]$ & \multicolumn{4}{|c|}{85} & \multicolumn{4}{|c|}{70} \\
\hline & & & $W_{2 a}[-]$ & \multicolumn{4}{|c|}{0.075} & \multicolumn{4}{|c|}{0.100} \\
\hline \multirow{3}{*}{ No. $2 b$} & \multirow{3}{*}{$\begin{array}{l}\text { heavy vehicle } \\
\text { above } 2 \text { axles }\end{array}$} & & 30 & 56 & 87.6 & 61 & 91.2 & 45 & 84.2 & 54 & 86.6 \\
\hline & & & $v_{2 b}[\mathrm{~km} / \mathrm{h}]$ & \multicolumn{4}{|c|}{85} & \multicolumn{4}{|c|}{70} \\
\hline & & S & $W_{2 b}[-]$ & \multicolumn{4}{|c|}{0.225} & \multicolumn{4}{|c|}{0.100} \\
\hline \multicolumn{4}{|c|}{$S P B I(\mathrm{~dB})$} & \multicolumn{2}{|c|}{$85.0^{1)}$} & \multicolumn{2}{|c|}{89.2} & \multicolumn{2}{|c|}{$78.9^{2)}$} & \multicolumn{2}{|c|}{82.2} \\
\hline
\end{tabular}

The measurements confirmed the expected increase in noise emissions assessed by $S P B I$ at sections of interest, at the motorway D3, and at the main road No. 61, as well. For MS1, higher values in the differences of the vehicle sound levels $4.8 \mathrm{~dB}\left(L_{1}\right), 4.3 \mathrm{~dB}\left(L_{2 a}\right)$, and $3.6 \mathrm{~dB}\left(L_{2 b}\right)$ were recorded between 2017 and 2008. The difference in the SPBI value for high driving speeds in the years 2017 and 2008 was $4.2 \mathrm{~dB}$. On MS1 motorway D3 during the 9-year period, a total of approximately 82 million vehicles passed in the monitored cross-section. The higher values of the vehicle sound levels and acoustic degradation of the surface on the D3 motorway are caused by the higher traffic loads, but also by higher reference speeds than those confirmed [21]. This was also reflected in the values of the reference $S P B I$ for the mean and high driving speeds. For MS2, the following differences in the reference values of the noise levels for the given measurement years are found between 2008 and 2020: $4.3 \mathrm{~dB}\left(L_{1}\right), 3.5 \mathrm{~dB}\left(L_{2 a}\right)$, and $2.4 \mathrm{~dB}\left(L_{2 b}\right)$. The SPBI difference at the mean driving speed in 2020 and 2008 was $3.3 \mathrm{~dB}$. On MS2 main road No. 61 during the 12-year period, a total of approximately 32 million vehicles passed in the monitored cross-section.

\section{Traffic noise degradation models}

Based on analysis of the evaluated $S P B I$ data for representative asphalt wearing courses of motorways and roads in Slovakia, the authors focused on finding correlations regarding the development of $S P B I$ values from the number of passes of all vehicles on the two monitored sections. For the number of passes $S\left[10^{6}\right]$, there was considered the traffic on the cross-section on both road sections.

For the sections of interest, the maximum noise levels were quantified at specific measurement temperatures, and the $S P B I$ indices calculated from them ( $\left.S P B I_{\text {measurement }}\right)$ were used to determine the power function as the optimal function for the correlation. For this type of equation, the highest value was found for the coefficient of determination corresponding to the physical logical dependence of the correlated characteristics. According to [20], vehicle sound pressure levels measured at air temperature $T_{i}\left[{ }^{\circ} \mathrm{C}\right]$ were adjusted to a reference temperature of $20{ }^{\circ} \mathrm{C}$. Therefore, a recalculation was performed according to Equation (2), used by the authors within the solution of the SPENS project, as follows:

$L_{e q}^{\wedge}\left(T=20^{\circ} \mathrm{C}\right)=L_{e q} \wedge\left(T_{i}\right)+k_{T} \cdot(T-20)$,

where $k_{T}=0.04$ and results of $S P B I$ indices were denoted as $S P B I_{S P E N S}$. The traffic noise degradation model with the highest value of the coefficient of determination was approximated by authors using the different value $k_{T}$ for different surfaces and results of $S P B I$ indexes were denoted as SPBI UNIZA.

The acoustic ageing of road surface SMA 11 can be expressed by the correlation of the changes in the SPBI on the road section MS1. Fig. 7 presents the dependence of the increase in SPBI 
values on the number of passes of all vehicles in one lane, considering the conversion to the air temperature coefficients $S P B I_{S P E N S}$ and $S P B I_{\text {UNIZA. }}$

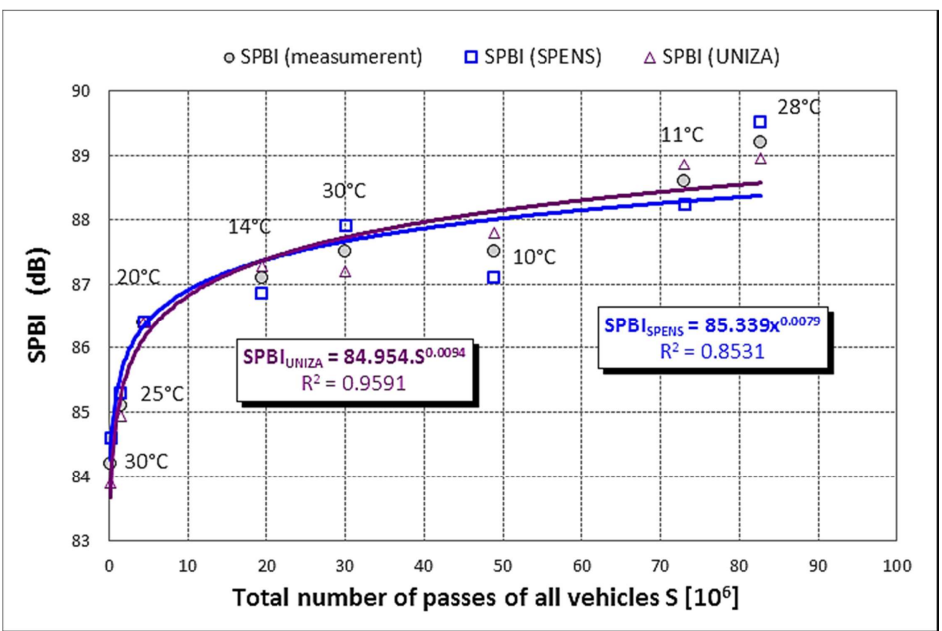

Fig. 7: Dependence of SPBI index of SMA 11 wearing course (MS1) on the number of all vehicle passes for the high driving speed in the sense EN ISO 11819-1.

The traffic noise degradation model of the SMA 11 road surface with the highest value of the coefficient of determination is approximated for the value $k_{T}=-0.03$ (SPBI UNIZA). In this case, the validity of the conversion factor used by the authors within the SPENS project solution $\left(k_{T, S P E N S}=0.04\right)$ is not confirmed; rather, the optimal conversion constant for the SMA 11 surface is found to be $k_{T, U N I Z A}=-0.03$.

Fig. 8 presents the acoustic ageing of road surface AC 11 as expressed by an increase in SPBI values, depending on the traffic load and a number of passages of vehicles $S\left[10^{6}\right]$ on MS2.

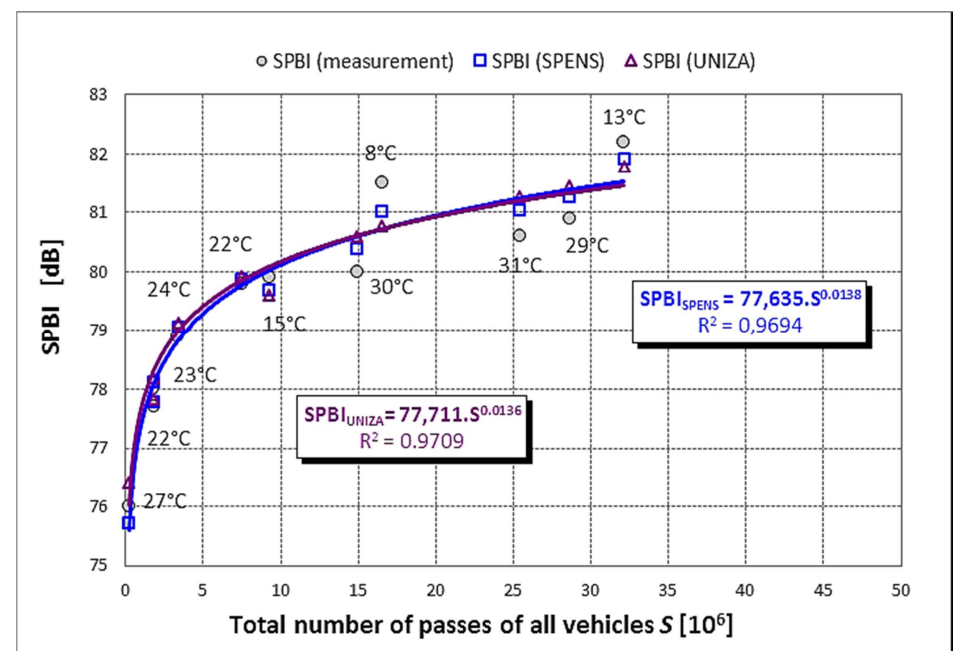

Fig. 8: Dependence of statistical pass-by index (SPBI) of wearing course AC 11 (MS2) on the number of all vehicle passes for the medium driving speed.

After an initial significant rise, the increase in $S P B /$ values gradually slows down. From a visual assessment of the dependencies in Fig. 8, it is clear that the differences in the coefficient $k_{T}$ are so small for surface AC 11 that their effects on the resulting values are practically negligible. The traffic noise degradation model of the AC 11 road surface with the highest value of the coefficient of determination is approximated for the value $k_{T}=0.06\left(S P B I_{\text {UNIZA }}\right)$. It follows that the validity of the conversion factor (used by the authors in the SPENS project) is confirmed.

The differences in the coefficient $k_{T}$ are more pronounced for the SMA 11 surface than for the AC 11 surface.

To compare the monitored surfaces of the asphalt road pavements in terms of acoustic degradation, the increment values of the sound level according to the total number of vehicle passes were also evaluated. Fig. 9 shows the linear dependence of the increment values on the logarithm of 
vehicle passes. Higher increment values at the selected number of passes were recorded at the AC11 surface. Thus, the AC 11 surface shows more pronounced acoustic ageing than the SMA11 surface. The differences in the traffic noise degradation models of the tested surfaces are shown. For the SMA 11 wearing course, the value of $1.0 \mathrm{~dB}$ in the SPBI represents the increase at 1.0 mil vehicle passes, and the value of $1.9 \mathrm{~dB}$ represents the increase from 1.0 mil to 10.0 mil vehicle passes and from 10.0 mil to 100.0 mil vehicle passes. For the $A C 11$ wearing course, at the equivalent vehicle passes, the increases are $1.3 \mathrm{~dB}$ and $2.5 \mathrm{~dB}$ in the $S P B I$, respectively. The SMA 11 wearing courses consist of coarse aggregate skeletons bound with mastic mortar (bitumen, sand, filler, and fibres), and are characterised by good resistance to permanent deformation, long-lasting skid resistance properties, and a long service life. The morphology of the SMA 11 surface, created by a dense gap-graded mixture, proved to be a surface with higher resistance to acoustic degradation.

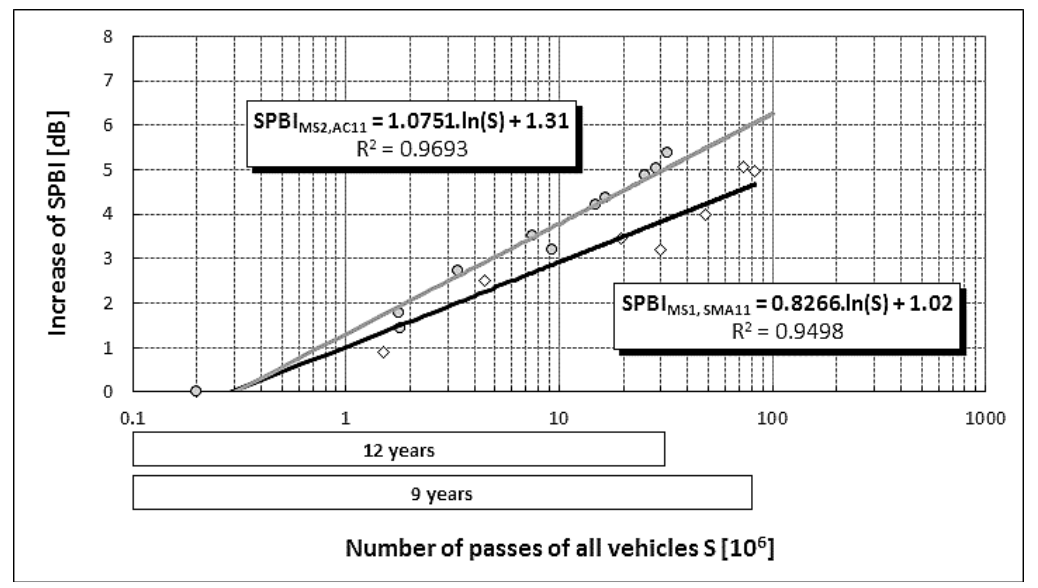

Fig. 9: Dependences of SPBI increase on the number of passes of all vehicles for a common type of wearing courses in the conditions of Central Europe, as evaluated for medium road speed category

(AC 11) and high road speed category (SMA 11).

The long-term noise measurements at the selected measuring sections showed acoustic ageing of the pavement surfaces.

\section{Noise mapping}

When assessing the noise exposure as part of road project investment or when creating strategic noise maps and action plans, a prediction of the noise exposure of the road environment can be processed through calculation models $[22,23]$. In these models, in the design period, an increase in the traffic load is considered, but without including the changes in the pavement surface characteristics owing to the gradual wear from traffic and climatic influences. The calculations consider a constant traffic speed, a constant traffic flow, a real 3D model of the road and its environment, and type of road pavement surface (asphalt or concrete). The example of the 3D model of road section MS2 is shown in Fig. 10.
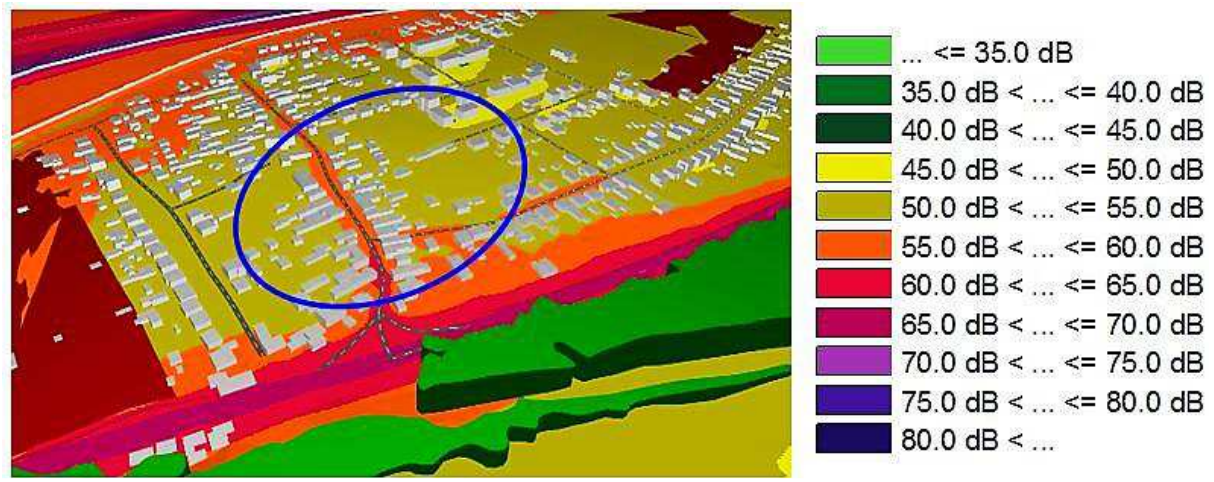

Fig. 10: 3D model of the road section MS2 in CadnaA software with measuring spot highlighting. 
At the time of preparing the article, the calculation method 'NMPB Routes 96' according to the French standard 'XPS 31-133' with application modification for the conditions of the Slovak Republic, was mandatory in the Slovak Republic for the creation of noise maps from road transport. Based on this methodology, the equivalent sound pressure level $L_{\text {Aeq,prediction }}$ was calculated in the CadnaA software and for illustration, the noise map of the road environment MS2 for the daytime was created, Fig. 11.
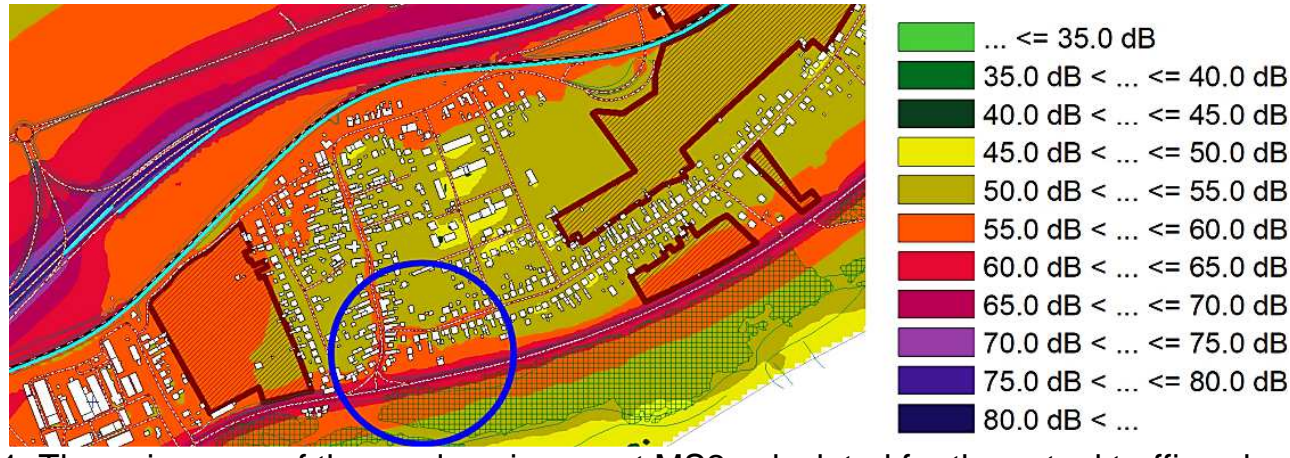

Fig. 11: The noise map of the road environment MS2 calculated for the actual traffic volume and composition.

Besides a maximum A-weighted sound levels $\left(L_{A \max }\right)$ measurement at the road section MS2 for SPBI determination purposes, there were simultaneously performed measurements of an equivalent sound pressure level $L_{\text {Aeq }}$ by a reputable accredited acoustic company with another sound analyser. After evaluating the noise measurements, a value of $72 \mathrm{~dB} L_{\text {Aeq,measured }}$ was obtained. That means that the direct measurements revealed $3 \mathrm{~dB}$ higher values than predicted by the model based on the actual number of vehicles. Taking into account the fact that the pavement is after those years in excellent condition without any distresses (according to diagnostics results as well as a visual inspection), these findings imply inappropriate corrections by which should be the ageing of a road pavement surface taking into consideration.

For the illustration purposes (Fig. 12) the model in the CadnaA software was adjusted by a correction of $+3 \mathrm{~dB}$, which was the difference between measured and predicted $L_{\text {Aeq }}$ values.
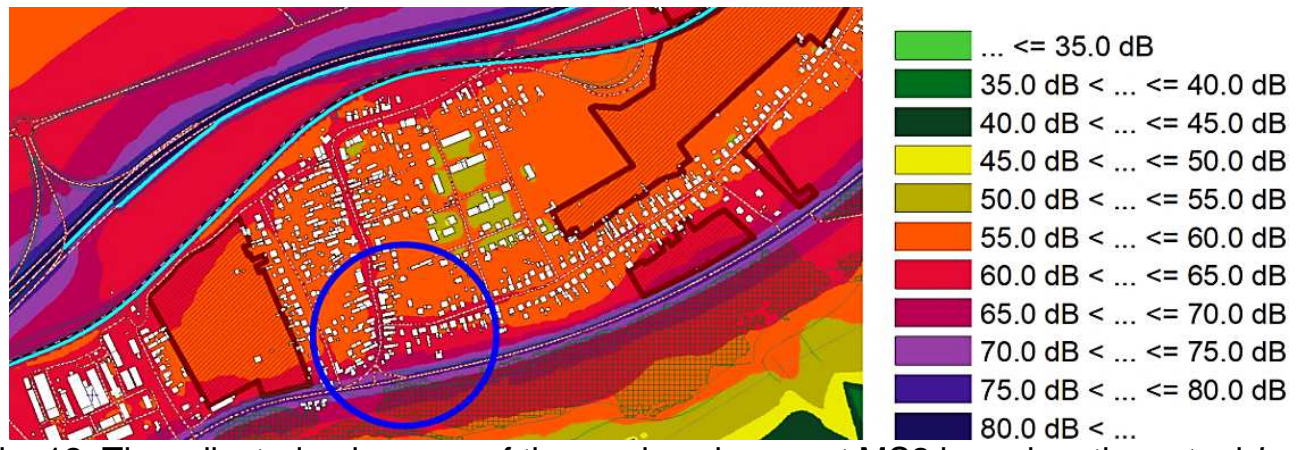

Fig. 12: The adjusted noise map of the road environment MS2 based on the actual $L_{\text {Aeq, }}$, measurements.

\section{Discussion}

The measurements over the period of 9 to 12 years confirmed the expected increase in noise emissions assessed by SPBI at both sections of interest, at the motorway D3 (MS1 - wearing course SMA 11) and at the main road No. 61 (MS2 - wearing course AC 11), as well. A valuable benefit of this study is that both wearing courses were located in the same climatic conditions and the impact of time and traffic volume could be investigated. Presented results of the change of noise level per year $0.42 \mathrm{~dB} /$ year of surface AC 11 (MS2) correspond with results in Danish/Californian comparative study of dense graded $A C$ surface $0.40 \mathrm{~dB} /$ year, respectively due to traffic volume as the change of noise level per 1 million vehicles $0.18 \mathrm{~dB} / 1$ mil vehicles of $A C 11$ surface (MS2) with results of DGAC surface $0.21 \mathrm{~dB} / 1$ mil vehicles. In contrast, the SMA 11 surface has a higher increase per year 0.55 
$\mathrm{dB} /$ year, and a lower increase of $0.06 \mathrm{~dB} / 1$ mil vehicles. This points to the fact that both the age of the surface as well as the traffic volume are relevant factors to take into consideration.

However, acoustic ageing is not the only one of the possible reasons that could cause the noise emissions increase. Another possible reason could be the changes in vehicles fleets over 9-12 years, while vehicles of all three categories might be heavier, stronger, and with different types of tyres. Considering the traffic flow speed (more than $60 \mathrm{~km} / \mathrm{h}$ ) at the road sections MS1 and MS2, and according to findings [24], from the two main sources of the traffic noise (the engine noise and the tyre/road surface interaction), only the rolling noise in combination with pavement surface changes can be taken into account.

While there hasn't been found any significant correlation between the tyre width and the noise level yet, there is no doubt that the influence of the type of tyre on tyre/road noise is undeniable. According to [25], CPX measurements showed the spread of the levels are 2-3 $d B(A)$ depending on tyre type on all road surfaces. According to [26], depending on the type of tyre the range of noise for the same pavement can be 6 to $8 \mathrm{~dB}$.

On the other hand, while the manufacturers over time try to design vehicles as well as tyres quieter the pavement surface ageing is unstoppable and thus the use of noise-reducing pavements should become the main goal for all national road authorities.

According to visual inspection and results of the regular diagnostics performed by the Slovak Road Administration, the values of longitudinal unevenness, rutting, and skid resistance prove that the road is in excellent condition. Thus, the insufficient road pavement maintenance and extreme degradation of the pavement surface (such as excessive ravelling, cracking, or other distresses) can be rejected as the next reason for the noise increase. This is the confirmation of the fact that there may not be necessarily a direct proportion between acoustic ageing and technical ageing of the road pavement surface.

In general, an increase of $3 \mathrm{~dB}$ can be considered as a doubling of the intensity (resp. double approximation) of the source of road traffic noise to the recipient. Fig. 12 shows the increase in the noise exposure of the road on MS2 and the negative impacts on its surroundings, which exceed the permissible values, Fig. 12. However, the long-term noise measurements on MS2 show an increase in the $S P B I$ of $5 \mathrm{~dB}$ for approximately 30 mil of all vehicle passes. Notably, the presented facts from the increase in the SPBI cannot be directly applied to the increase in $L_{\text {Aeg, }}$, but it is highly probable that the levels will reach similar increases as in the case of the increase in the SPBI.

These results could be adapted in models for assessing noise as part of road project investment, e.g., to create strategic noise maps and action plans by CNOSSOS-EU [19], or to provide economic appraisals of the environmental impacts (vehicle emissions, noise, and energy use) of new investment projects in road engineering in HDM-4.

\section{Conclusions}

In this study, the results from the long-term monitoring of two measuring sections, MS1 on motorway D3 and MS2 on main road No. 61, with different asphalt wearing courses (SMA 11 and AC 11), were investigated. By comparing the regressions of the maximum A-weighted sound level on the logarithm of the real vehicle velocity for vehicle categories $1,2 a$, and $2 b$ in the year 2008 (the starting year of traffic service on the section) and after 9 or 12 years of service, there was an increase in the sound level at all recorded speeds. Based on repeated noise measurements during the service of the monitored sections, the correlations were determined for the $S P B I$ indexes of the wearing courses SMA 11 and AC 11 on the number of all vehicle passes, and for high and medium driving speeds. The power function best describes the traffic noise degradation models, based on the dependence of the SPBI values on the total number of passes of all vehicles by approximation of the vehicle noise levels as adjusted to a reference temperature of $20{ }^{\circ} \mathrm{C}$, and using a conversion factor $k_{T}=0.06$ for road surface AC11 and $k_{T}=-0.03$ for surface SMA 11. The comparison of the increases revealed that the SMA11 surface had a higher resistance to acoustic degradation than the AC 11 wearing course. The presented outputs will be practically implemented in the creation of action projects to reduce traffic noise in Slovakia. They can also contribute to the refinement of input parameters for the prediction of noise from road traffic within CNOSSOS-EU 'Common Noise Assessment Methods in Europe'.

\section{Acknowledgments}

This publication was realized with the support of Operational Program Integrated Infrastructure 2014 - 2020 of the project: Innovative Solutions for Propulsion, Power and Safety Components of 
Transport Vehicles, code ITMS 313011V334, co-financed by the European Regional Development Fund.

\section{References}

[1] MELCER, J. - MERČIAKOVÁ, E. - PISCA, P.: Comparison of results obtained by two methods of surface mapping. Civil and environmental engineering, Vol. 16, Iss. 1, 2020, pp. 1-10, doi: 10.2478/cee-2020-0001.

[2] DECKÝ, M. - KOVÁČ, M. - MUŽíK, J. - MIČECHOVÁ, L. - ĎURIŠ, L.: Objectification of public bus stop's pavement surface morphology. Civil and environmental engineering, Vol. 14, Iss. 1/2018, pp. 44-53, doi: 10.2478/cee-2018-0006.

[3] FREITAS, E. F. - MARTINS, F. F. - OLIVEIRA, A. - SEGUNDO, I. R. - TORRES, H.: Traffic Noise and Pavement Distresses: Modelling and Assessment of Input Parameters Influence through Data Mining Techniques. Applied Acoustics, 138, 2018, doi:10.1016/j.apacoust.2018.03.019.

[4] SANDBERG, U. - EJSMONT, J. A.: Tyre/Road Noise Reference Book. 2002, ISBN 9163126109.

[5] VÁZQUEZ, V. F. - HIDALGO, M. E. - GARCÍA-HOZ, A. M. - CAMARA, A. - TERÁN, F. - RUIZTERAN, A. M. - PAJE, S. E.: Tire/Road Noise, Texture, and Vertical Accelerations: Surface Assessment of an Urban Road. Applied Acoustics, 160, 2020, doi:10.1016/j.apacoust.2019. 107153.

[6] LIPTAI, P. - MORAVEC, M. - LUMNITZER, E., et al.: Proposal of the sound insulating measures for a vibrational sorter and verification of the measured effectiveness. Advances in Science and Technology-Research Journal. Vol. 11, Iss. 3, 2017, pp. 196-203.

[7] LUMNITZER, E. - ANDREJIOVA, M. - BODNAROVA, A. G.: Verification of the impact oftheused type of excitation noise in determining the acoustic properties of separating constructions. Measurement. Vol. 78, 2016, pp. 83-89.

[8] LICITRA, G. - TETI, L. - CERCHIAI, M. - BIANCO, F.: The Influence of Tyres on the Use of the CPX Method for Evaluating the Effectiveness of a Noise Mitigation Action Based on Low-Noise Road Surfaces. Transportation Research Part D: Transport and Environment, 55, 2017, doi:10.1016/j.trd.2017.07.002.

[9] VAN BLOKLAND G. - TOLLENAAR C. H. - VAN LOON R.: Modelling of Acoustic Aging of Road Surfaces.QUESTIM: QUietness and Economics STimulate Infrastructure Management, Deliverable D2.2, CEDR Call 2012: Noise 2014.

[10] LICITRA, G. - MORO, A. - TETI, L. - DEL PIZZO, A. - BIANCO, F.: Modelling of Acoustic Ageing of Rubberized Pavements. Applied Acoustics, 146, 2019, doi:10.1016/j.apacoust.2018.11.009.

[11] ANFOSSO-LÉDÉE, F. - PICHAUD, Y.: Temperature Effect on Tyre-Road Noise. Applied Acoustics, 68, 2007, doi:10.1016/j.apacoust.2006.06.001.

[12] MARGORINOVA, M. - TROJANOVA, M. - DECKÝ, M. - REMIŠOVÁ, E.: Noise costs from road transport. Civil and environmental engineering, Vol. 14, Iss. 1, pp. 12-20, doi: 10.2478/cee-20180002.

[13] SANDBERG, U.: Time-Integrated Noise Reduction of Low Noise Pavements - Giving Fair Credit to the Most Efficient Pavements in the Long Term. Proceedings of INTERNOISE 2019, the 48th International Congress and Exhibition on Noise Control Engineering, 2019.

[14] DE LEÓN, G. - DEL PIZZO, A. - TETI, L. - MORO, A. - BIANCO, F. - FREDIANELLI, L. LICITRA, G.: Evaluation of Tyre/Road Noise and Texture Interaction on Rubberised and Conventional Pavements Using CPX and Profiling Measurements. Road Materials and Pavement Design, 21, 2020, doi:10.1080/14680629.2020.1735493.

[15] GARDZIEJCZYK, W. - GIERASIMIUK, P. - MOTYLEWICZ, M.: Noisiness of the Surfaces on Low-Speed Roads. Coatings, 6, 2016, doi:10.3390/coatings6020015.

[16] KLEIZIENÉ, R. - ŠERNAS, O. - VAITKUS, A. - SIMANAVIČIENÉ, R.: Asphalt Pavement Acoustic Performance Model. Sustainabiltiy, 11, 2019, doi:10.3390/su11102938.

[17] GARDZIEJCZYK, W.: The Effect of Time on Acoustic Durability of Low Noise Pavements - The Case Studies in Poland. Transportation Research Part D: Transport and Environment, 44, 2016, doi:10.1016/j.trd.2016.02.006.

[18] VAITKUS, A. - ANDRIEJAUSKAS, T. - VOROBJOVAS, V. - JAGNIATINSKIS, A. - FIKS, B. ZOFKA, E.:Asphalt Wearing Course Optimization for Road Traffic Noise Reduction. Construction and Building Materials, 152, 2017, doi:10.1016/j.conbuildmat.2017.06.130.

[19] KEPHALOPOULOS, S. - PSVIOTTI, M. - ANFOSSO-LÉDÉE, F.: Common Noise Assessment Methods in Europe (CNOSSOS-EU), 2012.

[20] STN EN ISO 1819-1:2002 Acoustics. Measurement of the Influence of Road Surfaces on Traffic Noise. Part 1: Statistical Pass-By Method (ISO 11819-1:1997). 
[21] YANG, W. - HE, J. - HE, C. - CAI, M.: Evaluation of Urban Traffic Noise Pollution Based on Noise Maps.Transportation Research Part D: Transport and Environment, 87, 2020, doi:10.1016/j.trd. 2020.102516.

[22] KIM, P. - RYU, H. - JEON, J. J. - CHANG, S. I.: Statistical Road-Traffic Noise Mapping Based on Elementary Urban Forms in Two Cities of South Korea. Sustainability, 13, 2021, doi.org/10.3390/ su13042365.

[23] SCHLOSSER T. - SCHLOSSER P. - HODAKOVA D. et al.: Modelling of Capacity and Public Transport Modal Split for New City Centre in Bratislava. In World Multidisciplinary Civil Engineering-Architecture-Urban Planning Symposium, WMCAUS 2019. Vol. 603, Iss. 5, 2019. Conference Proceedings, Materials Science and Engineering.

[24] DIJKINK J. H. - VAN KEULEN, W.: Silent Transport - An Innovative Silent Road Concept for Heavy Vehicles. Internoise 2004, Prague, Czech Republic, Paper No. 7672004.

[25] BERGE, T.: The Ranking of Rolling Noise from Passenger Car Tyres - a Comparison between Measurements and Modelling Results. The Journal of the Acoustical Society of America, 123, 2008, doi:10.1121/1.2935016.

[26] BENDTSEN, H. - GSPA K.: CEDR Technical Report 2017-01 State of the Art in Managing Road Traffic Noise: Noise-Reducing avements, 2017. 